N. Nakagoshi

Nagoya Math. J.

Vol. 73 (1979), 41-60

\title{
THE STRUCTURE OF THE MULTIPLICATIVE GROUP OF RESIDUE CLASSES MODULO $\mathfrak{p}^{N+1}$
}

\author{
NORIKATA NAKAGOSHI
}

\section{\$1. Introduction}

Let $k$ be an algebraic number field of finite degree and $p$ be a prime ideal of $k$, lying above a rational prime $p$. We denote by $G\left(p^{N+1}\right)$ the multiplicative group of residue classes modulo $\mathfrak{p}^{N+1}(N \geqq 0)$ which are relatively prime to $\mathfrak{p}$. The structure of $G\left(\mathfrak{p}^{N+1}\right)$ is well-known, when $N=0$, or $k$ is the rational number field $\boldsymbol{Q}$. If $k$ is a quadratic number field, then the direct decomposition of $G\left(\mathfrak{p}^{N+1}\right)$ is determined by A. Ranum [6] and F.H-Koch [4] who gives a basis of a group of principal units in the local quadratic number field according to H. Hasse [2]. In [5, Theorem 6.2], W. Narkiewicz obtains necessary and sufficient conditions so that $G\left(p^{N+1}\right)$ is cyclic, in connection with a group of units in the $p$ adic completion of $k$.

The structure of $G\left(p^{N+1}\right)$ is confirmed by that of the $p$-Sylow subgroup and the $p$-rank of $G\left(p^{N+1}\right)$ is given by T. Takenouchi [8]. If an algebraic number field $k$ contains a primitive $p$-th root of unity, the $p$-rank is also given by $\mathrm{H}$. Hasse [3, Teil $\left.I_{a}, \S 15\right]$.

In the present paper we shall establish the direct decomposition of $G\left(\mathfrak{p}^{N+1}\right)$ for each $N$ which gives another proof of T. Takenouchi's results $[8]$.

\section{§2. Notation and an outline of the investigation}

Let $e$ and $f$ be the ramification index and the degree of $\mathfrak{p}$ over $\boldsymbol{Q}$, respectively. Put $e_{1}=\left[\frac{e}{p-1}\right]$, where $[x]$ is the maximal integer $\leqq x$. We denote by $Z(m)$ a cyclic group of order $m$.

Let $H_{N+1}$ be the $(N+1)$-th unit group of the p-adic completion $k_{\mathfrak{p}}$ of $k$, that is,

\footnotetext{
Received October 3, 1977.
} 


$$
H_{N+1}=\left\{\eta \in k_{\mathfrak{p}} \mid \eta \equiv 1 \bmod \mathfrak{p}^{N+1}\right\} \quad(N=0,1, \cdots) .
$$

$H_{1}$ is called a group of principal units of $k_{p}$. Then one verifies easily that

$$
G\left(\mathfrak{p}^{N+1}\right) \cong Z\left(p^{f}-1\right) \times H_{1} / H_{N+1} \quad \text { (direct) },
$$

whence $H_{1} / H_{N+1}$ is isomorphic to the $p$-Sylow subgroup of $G\left(p^{N+1}\right)$.

Let $b_{N}(\nu)$ be a number of elements of a basis of $H_{1} / H_{N+1}$ whose orders are exactly $p^{\nu}(\nu \geqq 1)$. Then $H_{1} / H_{N+1}$ is expressed as direct product:

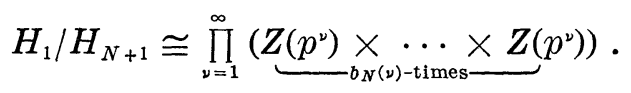

For our purpose it will suffice to establish a basis of $H_{1} / H_{N+1}$ for each $N \geqq 0$.

For any multiplicative group $G$ we denote by $G^{p^{\nu}}$ a subgroup of $G$ generated by $\sigma^{p^{\nu}}$ where $\sigma \in G$ and $\nu \geqq 1$. We define the $p$-rank $R_{N}$ of $G\left(p^{N+1}\right)$ by

$$
p^{R_{N}}=\left(G\left(\mathfrak{p}^{N+1}\right): G\left(\mathfrak{p}^{N+1}\right)^{p}\right) .
$$

$R_{N}$ will be given by Theorem 1 in $\S 3$.

We let $\pi$ be a prime element of $k_{p}$, fixed once for all. Put

$$
-p=\varepsilon \pi^{e},
$$

where $\varepsilon$ is a unit of $k_{p}$. Moreover, we let $\left\{\omega_{i}\right\}_{1 \leqq i \leqq f}$ be a system of representatives in $k_{\mathfrak{p}}$ for a basis of the residue class field modulo $\mathfrak{p}$ over the prime field.

Let $Z_{p}$ be the ring of $p$-adic integers. Then $H_{1}$ is a multiplicative $Z_{p}$-group and its system of generators over $Z_{p}$ is given by $H$. Hasse [2].

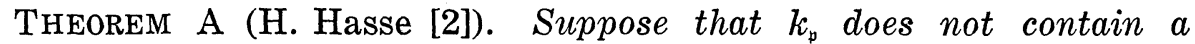
primitive p-th root of unity. Put

$$
\eta_{i s}=1+\omega_{i} \pi^{s} \quad\left(\begin{array}{l}
i=1, \cdots, f \\
1 \leqq s \leqq p e /(p-1), s \neq 0 \bmod p
\end{array}\right) .
$$

Then $\left\{\eta_{i s}\right\}$ is a $Z_{p}$-basis of $H_{1}$.

Let $\zeta_{\mu}$ be a primitive $p^{\mu}$-th root of unity for each $\mu \geqq 0$. Then we have 
THEOREM B (H. Hasse [2]). Suppose that $k_{\mathfrak{p}}$ contains $\zeta_{\mu}(\mu \geqq 1)$, but does not contain $\zeta_{\mu+1}$. Let $\lambda$ and $e_{0}$ be integers such that

$$
e=\varphi\left(p^{\lambda}\right) e_{0},
$$

where $\varphi$ is Euler's function and $e_{0}$ is prime to p. Put

$$
\begin{aligned}
& \eta_{i s}=1+\omega_{i} \pi^{s} \\
& \eta_{*}=1+\omega_{0} \pi^{e+e_{1}}
\end{aligned} \quad\left(\begin{array}{l}
i=1, \cdots, f \\
1 \leqq s \leqq e+e_{1}=p e /(p-1), s \neq 0 \bmod p
\end{array}\right),
$$

where $\omega_{1}, \cdots, \omega_{f}$ satisfy the following conditions:

$$
\omega_{1}^{p^{\lambda}}-\varepsilon \omega_{1}^{p \lambda-1} \equiv 0 \bmod \mathfrak{p}, \quad \omega_{i}^{p^{\lambda}}-\varepsilon \omega_{i}^{p^{\lambda-1}} \neq 0 \bmod \mathfrak{p}(2 \leqq i \leqq f)
$$

and $\omega_{0}$ is a unit of $k_{\mathfrak{p}}$ for which a congruence

$$
X^{p}-\varepsilon X \equiv \omega_{0} \bmod \mathfrak{p}
$$

has no solution $X$ in $k_{p}$.

Then $\left\{\eta_{i s}, \eta_{*}\right\}$ is a system of generators of $H_{1}$ over $Z_{p}$.

We note that $\lambda \geqq \mu$.

Now we sketch a plan to determine a basis of $H_{1} / H_{N+1}$. Let $\mu e+e_{1}$ $\leqq N<(\mu+1) e+e_{1}$ and $t \geqq 1$. Then we see by Lemma 7 in $\S 5$ that if $\mu=0, b_{t e+N}(\nu+t)=b_{N}(\nu)$; if $\mu \geqq 1, b_{t e+N}(\mu)=1+b_{N}(\mu-t), b_{t e+N}(\mu+t)$ $=b_{N}(\mu)-1$ and $b_{t e+N}(\nu+t)=b_{N}(\nu)$, where $\nu \neq \mu$ and $\nu+t \neq \mu$. Hence it is enough to compute $b_{N}(\nu)$ for $0 \leqq N<(\mu+1) e+e_{1}$.

We assume that $k_{p}$ contains $\zeta_{\mu}(\mu \geqq 0)$ but does not contain $\zeta_{\mu+1}$.

First suppose that $\mu=0$. Let $\eta_{i s} H_{N+1}$ be cosets of $H_{N+1}$ in $H_{1}$, where $\eta_{i s}$ are principal units defined by Theorem A. From Theorem A a system of canonical generators for $H_{1} / H_{N+1}$ is given by

$$
\left\{\eta_{i s} H_{N+1}\right\} \text {, }
$$

where $1 \leqq i \leqq f, 1 \leqq s \leqq \min (N, p e /(p-1))$ and $s \neq 0 \bmod p$. Let $g_{N}(\nu)$ be a number of generators of (2) such that $\eta_{s s}^{p^{\nu}} \equiv 1 \bmod p^{N+1}$. In $\S 5$ we shall prove

$$
g_{N}(1)+\sum_{\nu=2}^{\infty} \nu\left(g_{N}(\nu)-g_{N}(\nu-1)\right)=N f
$$

(see (17) in $\S 5$ ), hence (2) is a basis of $H_{1} / H_{N+1}$. Then $b_{N}(\nu)$ are given as follows: 


$$
\left\{\begin{array}{l}
b_{N}(1)=g_{N}(1), \\
\ddot{b_{N}(\nu)}=g_{N}(\nu)-g_{N}(\nu-1) \quad(\nu \geqq 2) .
\end{array}\right.
$$

Furthermore, we shall compute orders $p^{\nu(N: i, s)}$ of $\eta_{i s}$ modulo $\mathfrak{p}^{N+1}$, using Corollary 8 in $\S 5$. Then we can determine a basis of $H_{N+1}$ for each $N$ (see Proposition 11 in $\S 5$ ). Since a basis of $H_{1}$ is given by Theorem A, the direct decomposition of $H_{1} / H_{N+1}$ is easily obtained.

Secondly we assume $\mu \geqq 1$. Put

$$
\begin{aligned}
S=\left\{(i, s) \mid 1 \leqq i \leqq f, 1 \leqq s \leqq e+e_{1}=p e_{1},\right. & \\
s & \left.\neq 0 \bmod p,(i, s) \neq\left(1, e_{0}\right)\right\} .
\end{aligned}
$$

The number of elements of $S$ is equal to $(e f-1)$. If $\lambda=\mu$, then $\eta_{1 e_{0}}$ $=\zeta_{\mu}$ and $\left\{\eta_{*}, \eta_{i s}\right\}_{(i, s) \in S}$ is a $Z_{p}$-basis of $H_{1}([2$, p. 232]). If $\lambda>\mu$, then we observe by $[2$, p. 231] that

$$
\eta_{1 e_{0}}^{p \lambda-\mu}=\zeta_{\mu} \cdot \eta_{*}^{\beta_{*}} \prod_{(i, s) \in S} \eta_{i s}^{\beta_{i s}},
$$

where $\beta_{*}$ and $\beta_{i s}$ are $p$-adic integers. Let $H_{01}$ be a multiplicative $\boldsymbol{Z}_{p^{-}}$ group generated by $\left\{\eta_{*}, \eta_{i s}\right\}_{(i, s) \in S}$. Then by [2, p. 230] we have a direct decomposition of $H_{01}$ :

$$
H_{01}=\left\langle\eta_{*}\right\rangle \times \prod_{(i, s) \in S}\left\langle\eta_{i s}\right\rangle \quad \text { (direct), }
$$

where $\langle\eta\rangle$ stands for a cyclic group generated by $\eta$.

Let $\eta_{*} H_{N+1}, \eta_{i s} H_{N+1}$ be cosets of $H_{N+1}$ in $H_{1}$ and $p^{\nu(N: *)}, p^{\nu(N: i, s)}$ be their orders in $H_{1} / H_{N+1}$, respectively. From Theorem $\mathrm{B}$ we have a system of canonical generators for $H_{1} / H_{N+1}$ as follows:

$$
\begin{gathered}
\left\{\eta_{i s} H_{N+1}\right\}, \quad \text { if } 1 \leqq N<e+e_{1}, \\
\left\{\eta_{*} H_{N+1}, \eta_{i s} H_{N+1}\right\}, \quad \text { if } e+e_{1} \leqq N,
\end{gathered}
$$

where $1 \leqq i \leqq f, 1 \leqq s \leqq \min \left(N, e+e_{1}\right)$ and $s \equiv 0 \bmod p$. Let $g_{N}(\nu)$ be a number of generators defined by $\left(8_{1}\right)$ or $\left(8_{2}\right)$ such that $\eta_{i s}^{p v} \equiv 1 \bmod p^{N+1}$, $\eta_{*}^{p^{\nu}} \equiv 1 \bmod \mathfrak{p}^{N+1}$. Then $\left(8_{1}\right)$ or $\left(8_{2}\right)$ is a basis of $H_{1} / H_{N+1}$ if and only if the equality (3) holds. It will be proved by (17) in $\S 5$ that (i) $\left(8_{1}\right)$ is a basis of $H_{1} / H_{N+1}$, (ii) $\left(8_{2}\right)$ is a basis of $H_{1} / H_{N+1}$ if and only if $\nu\left(N: 1, e_{0}\right)$ $=\lambda$. If the equality (3) holds, then $b_{N}(\nu)$ are given by (4).

If $N \geqq e+e_{1}$ and $\nu\left(N: 1, e_{0}\right) \neq \lambda$, then it will be possible to determine a basis of $H_{N+1}$ (see Proposition 11 in $\S 5$ ) and we observe that 
$H_{N+1}$ is a subgroup of $H_{01}$. Hence we can find a relation between $\eta_{*}$, $\eta_{1 e_{0}}$ and $\eta_{i s}$ modulo $\mathfrak{p}^{N+1}$ (see (18) in $\S 6$ ) which is induced by (6). Let $Z$ be the ring of rational integers. Let $M$ be a free $Z$-module generated by $\tilde{\eta}_{*}, \tilde{\eta}_{1 e_{0}}$ and $\tilde{\eta}_{i s}((i, s) \in S)$. Let $\psi: M \rightarrow H_{1} / H_{N+1}$ be a homomorphism defined by $\psi\left(\tilde{\eta}_{*}\right) \equiv \eta_{*} \bmod \mathfrak{p}^{N+1}, \psi\left(\tilde{\eta}_{1 e_{0}}\right) \equiv \eta_{1 e_{0}} \bmod \mathfrak{p}^{N+1}$ and $\psi\left(\tilde{\eta}_{i s}\right) \equiv \gamma_{i s} \bmod \mathfrak{p}^{N+1}$. Then we shall have a system of canonical generators for $\operatorname{Ker} \psi$. Hence the direct decomposition of $H_{1} / H_{N+1} \cong M / \operatorname{Ker} \psi$ will be obtained using elementary divisors of a certain matrix (see (9) of Theorem 3) whose entries are $p^{\nu(N: i, s)}, p^{\nu(N: *)}$ and $p$-components of exponents appearing in the relation (18) in $\S 6$.

\section{§3. Theorems}

We shall prove the following assertions:

THEOREM 1 (cf. [3] and [8]). The p-rank $R_{N}$ of $G\left(\mathfrak{p}^{N+1}\right)$ is given by

$$
R_{N}= \begin{cases}\left(N-\left[\frac{N}{p}\right]\right) f, & \text { if } 0 \leqq N<e+e_{1}, \\ e f, & \text { if } N \geqq e+e_{1} \text { and } k_{p} \ni \zeta_{1}, \\ e f+1, & \text { if } N \geqq e+e_{1} \text { and } k_{p} \ni \zeta_{1} .\end{cases}
$$

THEOREM 2. Suppose that $k_{\mathfrak{p}}$ does not contain $\zeta_{1}$. Let $0 \leqq N \leqq e+e_{1}$. Then it follows that for each $t \geqq 0$

$$
\begin{aligned}
G\left(p^{t e+N+1}\right) \cong & \left.Z\left(p^{f}-1\right) \times \prod_{\nu=1}^{\infty}(\underbrace{Z\left(p^{\nu+t}\right) \times \ldots \times Z\left(p^{\nu+t}\right)})\right) \\
& \times(\underbrace{Z\left(p^{t}\right) \times \ldots \times}_{b_{N}(\nu) \text { times }} \times R_{\left(e+N-R_{N}\right) \text {-times }} Z\left(p^{t}\right))
\end{aligned}
$$

where $R_{t e+N}, R_{N}$ are p-ranks of $G\left(\mathfrak{p}^{t e+N+1}\right), G\left(\mathfrak{p}^{N+1}\right)$, respectively, and

$$
b_{N}(\nu)=\left(\left[\frac{N}{p^{\nu-1}}\right]-2\left[\frac{N}{p^{\nu}}\right]+\left[\frac{N}{p^{\nu+1}}\right]\right) f .
$$

THEOREM 3. Suppose that $k_{\mathfrak{p}}$ contains $\zeta_{\mu}(\mu \geqq 1)$ but does not contain $\zeta_{\mu+1}$. Let $\lambda$ and $e_{0}$ be as in Theorem B. Then the direct decomposition of $G\left(\mathfrak{p}^{N+1}\right)$ is expressed as follows:

( I ) In the case where $1 \leqq N<e+e_{1}$,

$$
G\left(\mathfrak{p}^{N+1}\right) \cong Z\left(p^{f}-1\right) \times \prod_{\nu=1}^{\infty}(\underbrace{Z\left(p^{\nu}\right)} \underset{b_{N}(\nu) \text {-times }}{\times \ldots Z} \times\left(p^{\nu}\right)),
$$

where $b_{N}(\nu)$ are equal to those of Theorem 2. 
(II) In the case where $e+e_{1} \leqq N<(\mu+1) e+e_{1}$ and $\nu\left(N: 1, e_{0}\right)$ $=\lambda$,

$$
G\left(\mathfrak{p}^{N+1}\right) \cong Z\left(p^{f}-1\right) \times \prod_{\nu=1}^{\infty}(\underbrace{Z\left(p^{\nu}\right)} \underset{b_{N}(\nu)-\text { times }}{\times} \times Z\left(p^{\nu}\right)) ;
$$

$b_{N}(\nu)$ are given as follows:

Let $a$ be a rational integer $(1 \leqq a \leqq \mu)$ such that ae $+e_{1} \leqq N$ $<(a+1) e+e_{1}$.

For $\nu \leqq a-1, b_{N}(\nu)=0$.

For $\nu=a, b_{N}(a)=\left((a+1) e-N+\left[\frac{N-a e}{p}\right]\right) f+\beta_{N}(a)$.

For $\nu \geqq a+1$,

$$
\begin{aligned}
b_{N}(\nu)= & \left(\left[\frac{N-(a+\delta-1) e}{p^{\nu-a-\delta}}\right]-2\left[\frac{N-(a+\delta-1) e}{p^{\nu-a-\delta+1}}\right]\right. \\
& \left.+\left[\frac{N-(a+\delta-1) e}{p^{\nu-a-\delta+2}}\right]\right) f+\beta_{N}(\nu),
\end{aligned}
$$

where

$$
\beta_{N}(a)=\left\{\begin{array}{ll}
2, & \text { if } a=\lambda=\mu, \\
1, & \text { if } a \neq \lambda,
\end{array} \quad \beta_{N}(\nu)= \begin{cases}1, & \text { if } \nu=\lambda \geqq a+1 \\
-1, & \text { if } \nu=\lambda+a, \\
0, & \text { otherwise }(\nu \geqq a+1)\end{cases}\right.
$$

and

$$
\delta= \begin{cases}0, & \text { if } N=a e+e_{1}, \\ 1, & \text { if } a e+e_{1}<N<(a+1) e+e_{1} .\end{cases}
$$

(III) In the case where $e+e_{1}<N<(\mu+1) e+e_{1}$ and $\nu\left(N: 1, e_{0}\right)$ $>\lambda$, there exists a rational integer $a(1 \leqq a \leqq \mu)$ such that ae $+e_{1} \leqq N$ $<(a+1) e+e_{1}$. Let $p^{a} i_{\text {is }}$ be p-components of $\beta_{i s} p^{\mu}$ where $\beta_{i s}$ are p-adic integers defined by (6). Put

$$
a_{i s}=\min \left\{\nu(N: i, s), a_{i s}^{\prime}\right\} \quad \text { for }(i, s) \in S,
$$

where $S$ is given by (5). If $N=a e+e_{1}$ and $\left(e+e_{1}\right) / p^{\nu-a+1}<s$ $\leqq\left(e+e_{1}\right) / p^{\nu-a}$, then $\nu(N: i, s)=\nu \geqq a ;$ if $a e+e_{1}<N<(a+1) e+e_{1}$ and $(N-a e) / p^{\nu-a}<s \leqq(N-a e) / p^{\nu-a-1}$, then $\nu(N: i, s)=\nu \geqq a$. Let $p^{c_{0}}, p^{c_{1}}, \cdots, p^{c_{e f}}$ be elementary divisors of the following $(e f+2) \times(e f+1)$ matrix 
(9)

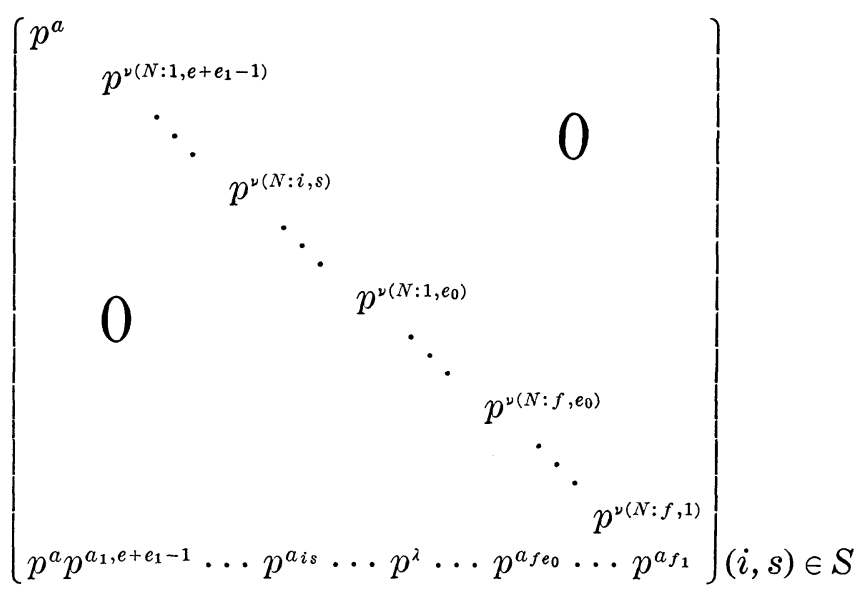

It then follows that

$$
G\left(p^{N+1}\right) \cong Z\left(p^{f}-1\right) \times Z\left(p^{c_{0}}\right) \times Z\left(p^{c_{1}}\right) \times \cdots \times Z\left(p^{c_{e f}}\right) .
$$

(IV) In the case where $\mu e+e_{1} \leqq N<(\mu+1) e+e_{1}$, we let $G\left(p^{N+1}\right)$ be of type $\left(p^{f}-1, p^{\mu}, p^{d_{1}}, \cdots, p^{d_{e f}}\right)$ which is determined by (II) and (III). Then $G\left(p^{t e+N+1}\right)$ is of type $\left(p^{f}-1, p^{\mu}, p^{d_{1}+t}, \cdots, p^{d_{e f}+t}\right)$ for each $t \geqq 0$.

Remarks. Under the hypothesis of Theorem 3 (i) if $\lambda=\mu$ and $N$ $\geqq e+e_{1}$, then $\nu\left(N: 1, e_{0}\right)=\lambda$ (cf. [2, p. 216]); (ii) if $N=a e+e_{1}$, then $\lambda \leqq \nu\left(N: 1, e_{0}\right) \leqq \lambda+a-1$; (iii) if $a e+e_{1}<N<(a+1) e+e_{1}$, then $\lambda$ $\leqq \nu\left(N: 1, e_{0}\right) \leqq \lambda+a$ (cf. proof of Corollary 10 of $\S 5$ ); (iv) if $N \geqq \mu e+e_{1}$, then $H_{N+1}$ is a subgroup of a free part of $H_{1}$.

COROLlaRY 4. If $\mathfrak{p}$ is an unramified prime ideal of $k$, lying above a rational prime $p$, then we have

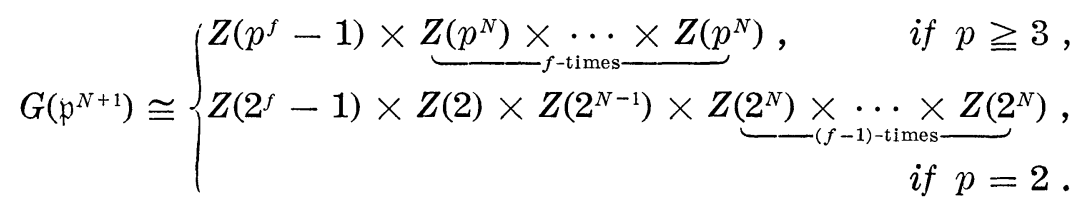

\section{§4. Proof of Theorem 1}

It follows from (1) that

LEMMA 5 (cf. [2, p. 220] and [3, Teil $\left.\left.I_{a}, \S 15\right]\right)$. Let $\gamma$ be an integer of $k_{p}$. Then 


$$
\left(1+\gamma \pi^{s}\right)^{p} \equiv \begin{cases}1+\gamma^{p} \pi^{p s} \bmod p^{p s+1}, & \text { if } 1 \leqq s<e /(p-1), \\ 1+\left(\gamma^{p}-\varepsilon \gamma\right) \pi^{p s} \bmod p^{p s+1}, & \text { if } s=e /(p-1), \\ 1-\varepsilon \gamma \pi^{s+e} \bmod p^{s+e+1}, & \text { if if } s>e /(p-1) .\end{cases}
$$

Now we shall prove Theorem 1 . First we note that $k_{p}$ contains a primitive $p$-th root of unity if and only if $e \equiv 0 \bmod (p-1)$ and a congruence

$$
X^{p}-\varepsilon X \equiv 0 \bmod \mathfrak{p}
$$

has a solution $X \neq \equiv 0 \bmod \mathfrak{p}$ in $k_{\mathfrak{p}}$ (cf. [2, p. 215]).

According to H. Hasse [3], we shall use the following notation:

$\alpha$ : a number of $k_{\mathfrak{p}}$, prime to $\mathfrak{p}$.

$\gamma:$ an integer of $k_{p}$.

$\gamma_{0}:$ an integer of $k_{\mathfrak{p}}$ such that $\gamma_{0} \equiv 0 \bmod \mathfrak{p}$.

$\eta$ : a principal unit of $k_{p}$.

$\mu_{s}:$ an integer of $k_{\mathfrak{p}}$ such that $\mu_{s} \equiv \alpha^{p} \bmod \mathfrak{p}^{s}(s \geqq 1)$.

$\alpha_{s}:$ an integer of $k_{\mathfrak{p}}$ such that $\alpha_{s}^{p} \equiv 1 \bmod \mathfrak{p}^{s}$.

$\gamma_{s}:$ an integer of $k_{\mathfrak{p}}$ such that

$$
\alpha_{s}^{p} \equiv 1+\gamma_{s} \pi^{s} \bmod \mathfrak{p}^{s+1} .
$$

Each of these notations stands for a general element of a group, but will sometimes be used to stand for the group itself. The $p$-rank $R_{N}$ of $G\left(p^{N+1}\right)$ is then given by

$$
\begin{aligned}
p^{R_{N}} & =\left(G\left(\mathfrak{p}^{N+1}\right): G\left(\mathfrak{p}^{N+1}\right)^{p}\right)=\left(\alpha: \mu_{N+1}\right) \\
& =\left(\alpha: \mu_{1}\right)\left(\mu_{1}: \mu_{2}\right) \cdots\left(\mu_{N}: \mu_{N+1}\right)
\end{aligned}
$$

and we have

$$
\left(\mu_{s}: \mu_{s+1}\right)=\left(\gamma: \gamma_{s}\right) \quad(1 \leqq s \leqq N) .
$$

It will be verified that

$$
\left(\alpha: \mu_{1}\right)=1 \text {, }
$$

(c)

$$
\begin{gathered}
\left(\mu_{s}: \mu_{s+1}\right)= \begin{cases}1, & \text { if } 1 \leqq s<e+e_{1} \text { and } s \equiv 0 \bmod p, \\
p^{s}, & \text { if } 1 \leqq s<e+e_{1} \text { and } s \neq 0 \bmod p,\end{cases} \\
\left(\mu_{e+e_{1}}: \mu_{e+e_{1}+1}\right)= \begin{cases}1, & \text { if } e \equiv 0 \bmod (p-1) \text { and } k_{\mathfrak{p}} \ni \zeta_{1}, \\
p, & \text { if } k_{\mathfrak{p}} \ni \zeta_{1}, \\
p^{f}, & \text { if } e \neq 0 \bmod (p-1),\end{cases} \\
\left(\mu_{s}: \mu_{s+1}\right)=1, \quad \text { if } s>e+e_{1} .
\end{gathered}
$$


Proof of $(a)$. Since $\left(\alpha: \mu_{1}\right)=\left(\alpha: \alpha^{p} \eta\right)$ is a power of $p$ and $\alpha / \gamma$ is a cyclic group of order $\left(p^{f}-1\right),\left(\alpha: \mu_{1}\right)=1$.

Proof of $(b),(c)$ and $(d)$. Since $\alpha_{s}^{p} \equiv 1 \bmod \mathfrak{p}$ and the order of $G(p)$ is equal to $p^{f}-1$ which is prime to $p, \alpha_{s} \equiv 1 \bmod \mathfrak{p}$. If $\alpha_{s}=1$, then by (10) we see that $\gamma_{s} \equiv 0 \bmod \mathfrak{p}$. Let $\alpha_{s} \neq 1$. We can put

$$
\alpha_{s}=1+\varepsilon_{s} \pi^{\tilde{s}},
$$

where $\bar{s} \geqq 1$ and $\varepsilon_{s}$ is a unit of $k_{p}$. Then it follows from Lemma 5

$$
\alpha_{s}^{p} \equiv \begin{cases}1+\varepsilon_{s}^{p} \pi^{p \bar{s}} \bmod \mathfrak{p}^{p \bar{s}+1}, & \text { if } 1 \leqq \bar{s}<e /(p-1), \\ 1+\left(\varepsilon_{s}^{p}-\varepsilon \varepsilon_{s}\right) \pi^{p \bar{s}} \bmod \mathfrak{p}^{p \bar{s}+1}, & \text { if } \bar{s}=e /(p-1), \\ 1-\varepsilon \varepsilon_{s} \pi^{\bar{s}+e} \bmod \mathfrak{p}^{\bar{s}+e+1}, & \text { if } \bar{s}>e /(p-1) .\end{cases}
$$

If $1 \leqq s<e+e_{1}$ and $s \equiv 0 \bmod p$, then by (10) $\gamma_{s}$ modulo $p$ contains $\left(\varepsilon_{s}^{p}+\gamma_{0}\right)$ modulo $p$. Hence $\left(\gamma: \gamma_{s}\right)=1$, because of $\left(\gamma: \gamma_{s}\right) \leqq\left(\gamma: \varepsilon_{s}^{p}+\gamma_{0}\right)=1$.

Suppose that $1 \leqq s<e+e_{1}$ and $s \neq 0 \bmod p$. Then from the above congruences and (10) we can conclude that

$$
\begin{cases}\gamma_{s} \equiv 0 \bmod \mathfrak{p}, & \text { if } 1 \leqq \bar{s}<e /(p-1) \text { and } s<p \bar{s}, \\ \varepsilon_{s}^{p} \pi^{p \bar{s}} \equiv 0 \bmod \mathfrak{p}^{p \bar{s}+1}, \text { a contradiction, }, & \text { if } s>p \bar{s} \\ \gamma_{s} \equiv 0 \bmod \mathfrak{p}, & \text { if } \bar{s} \geqq e /(p-1) .\end{cases}
$$

Hence we have $\left(\gamma: \gamma_{s}\right)=\left(\gamma: \gamma_{0}\right)=p^{f}$ which shows (b) by (12).

Let $s=e+e_{1}$. Using the above congruences and (10) we see that

$$
\begin{cases}\varepsilon_{s}^{p} \pi^{p \bar{s}} \equiv 0 \bmod \mathfrak{p}^{p \bar{s}+1}, \text { a contradiction }, & \text { if } 1 \leqq \bar{s}<e /(p-1) \\ \gamma_{s} \equiv \varepsilon_{s}^{p}-\varepsilon \varepsilon_{s} \bmod \mathfrak{p}, & \text { if } \bar{s}=e /(p-1), \\ \gamma_{s} \equiv 0 \bmod \mathfrak{p}, & \text { if } \bar{s}>e /(p-1) .\end{cases}
$$

If $k_{p} \ni \zeta_{1}$, then $\gamma / \gamma_{0}^{\prime} \cong\left(\left(\gamma^{p}-\varepsilon \gamma\right)+\gamma_{0}\right) / \gamma_{0}$, where $\gamma_{0}^{\prime}$ are solutions of $X^{p}-$ $\varepsilon X \equiv 0 \bmod \mathfrak{p}$, and $\left(\gamma: \gamma_{0}\right) /\left(\gamma: \gamma_{0}^{\prime}\right)=p$. Hence $\left(\gamma: \gamma_{s}\right)=\left(\gamma:\left(\gamma^{p}-\varepsilon \gamma\right)+\gamma_{0}\right)=$ $p$. If $e \equiv 0 \bmod (p-1)$ and $k_{\mathfrak{p}} \supsetneqq \zeta_{1}$, then $\gamma_{s} \equiv \varepsilon_{s}^{p}-\varepsilon \varepsilon_{s} \neq 0 \bmod \mathfrak{p} \operatorname{and}\left(\gamma: \gamma_{s}\right)$ $=1$. If $e \neq 0 \bmod (p-1)$, then $\left(\gamma: \gamma_{s}\right)=\left(\gamma: \gamma_{0}\right)=p^{f}$. Therefore (c) is obtained by (12).

Assume that $s>e+e_{1}$. Then we have by Lemma 5

$$
\left(1+\gamma \pi^{s-e}\right)^{p} \equiv 1-\varepsilon \gamma \pi^{s} \bmod \mathfrak{p}^{s+1} .
$$

Hence by (10) $\gamma_{s}$ modulo $\mathfrak{p}$ contains $\left(-\varepsilon \gamma+\gamma_{0}\right)$ modulo $\mathfrak{p}$ and $\left(\gamma: \gamma_{s}\right)$ $=\left(\gamma:\left(-\varepsilon \gamma+\gamma_{0}\right)\right)=1$, thereby proving (d). By (11) and (12) we have Theorem 1. 
For instance, we compute $R_{N}$ when $N \geqq e+e_{1}$ and $e \neq 0 \bmod (p-1)$. Put $e=(p-1) e_{1}+r, 1 \leqq r \leqq p-2$. Then by (11), (a), (b), (c) and (d) we have

$$
\begin{aligned}
R_{N} & =\left(e+e_{1}-1-\left[\frac{e+e_{1}-1}{p}\right]\right) f+f \\
& =\left(e+e_{1}-1-\left[e_{1}+\frac{r-1}{p}\right]\right) f+f=e f .
\end{aligned}
$$

\section{§5. Preliminaries to the proof of Theorem 2 and Theorem 3}

In order to prove Theorem 2 and Theorem 3 we need some results which we obtain in this section. Throughout this section we assume that $k_{\mathfrak{p}}$ contains $\zeta_{\mu}(\mu \geqq 0)$ but does not contain $\zeta_{\mu+1}$.

The following proposition is well-known:

Proposition 6 (cf. [2, §15] and [5, Chap. V]). If $N \geqq e_{1}$, then $H_{N+1}$ is a free $Z_{p}$-group and $H_{N+1} \cong H_{e+N+1}$ by $\eta \rightarrow \eta^{p}\left(\eta \in H_{N+1}\right)$.

LEMMA 7. Suppose that $N \geqq e_{1}$ and $H_{N+1}$ is a subgroup of a $Z_{p}$-free part $\overline{H_{01}}$ of $H_{1}$. Let $H_{1} / H_{N+1}$ be of type $\left(p^{s_{0}}, p^{s_{1}}, \cdots, p^{s_{e f}}\right)$. Then we can take $s_{0}=\mu$ and $H_{1} / H_{t e+N+1}$ is of type $\left(p^{s_{0}}, p^{s_{1}+t}, \cdots, p^{s_{e f}+t}\right)$ for each $t \geqq 0$.

Remark. In Lemma 7 we allow that $s_{j}=0(0 \leqq j \leqq e f)$.

Proof. We have an expression of $H_{1}$ as direct product (cf. [2, p. 222]):

$$
H_{1}=\left\langle\zeta_{\mu}\right\rangle \times \overline{H_{01}},
$$

where $\left\langle\zeta_{\mu}\right\rangle$ is a cyclic group generated by $\zeta_{\mu}$ and $\overline{H_{01}}$ is of rank $e f$. By the hypothesis of the Lemma 7 we have

$$
H_{1} / H_{N+1} \cong\left\langle\zeta_{\mu}\right\rangle \times \overline{H_{01}} / H_{N+1} \quad \text { (direct). }
$$

Hence there exists a $Z_{p}$-basis $\left\{\eta_{1}, \cdots, \eta_{e f}\right\}$ of $\overline{H_{01}}$ such that $\left\{\eta_{1}^{p_{1}}, \cdots, \eta_{e f}^{p_{e f}}\right\}$ is a $Z_{p}$-basis of $H_{N+1}$. It then follows from Proposition 6 that $\left\{\eta_{1}^{p_{1}{ }^{1+1}}\right.$, $\left.\cdots, \eta_{e f}^{p_{e f} e^{+1}}\right\}$ is a $Z_{p}$-basis of $H_{e+N+1}$. Thus the Lemma 7 is proved by induction. q.e.d.

If $\mu=0$ and $N \geqq e_{1}$, then we observe by Lemma 7 that $b_{t e+N}(\nu+t)$ $=b_{N}(\nu)$ for each $t \geqq 0$. Hence all $G\left(\mathfrak{p}^{N+1}\right)$ are determined by factor groups $H_{1} / H_{1}, \cdots, H_{1} / H_{e+e_{1}}$. If $\mu \geqq 1$ and $N \geqq \mu e+e_{1}$, then $H_{N+1}$ is a subgroup of $H_{1}^{p^{\mu}}=\left\{\eta^{p^{\mu}} \mid \eta \in H_{1}\right\}$. Hence $H_{N+1}$ is a subgroup of a free part of $H_{1}$. In this case for each $t \geqq 1$ it follows that $b_{t e+N}(\mu)=1+b_{N}(\mu-t)$, 
$b_{t e+N}(\mu+t)=b_{N}(\mu)-1$ and $b_{t e+N}(\nu+t)=b_{N}(\nu)$, where $\nu \neq \mu$ and $\nu+t$ $\neq \mu$. Hence all $G\left(\mathfrak{p}^{N+1}\right)$ are determined by factor groups $H_{1} / H_{2}, \cdots$, $H_{1} / H_{\mu e+e_{1}}$.

In order to compute $g_{N}(\nu), \nu(N: i, s)$ and $\nu(N: *)$ defined in $\S 2$ we need the following corollary to Lemma 5 (cf. [7] and [9, Corollary 1.2]):

COROLlaRY 8. Let $\eta$ be an element of $k_{\mathfrak{p}}$ such that $\eta \equiv 1 \bmod \mathfrak{p}^{s}$ and $\eta \equiv 1 \bmod p^{s+1}(s \geqq 1)$. Let $\tau$ be the least non-negative integer such that $p^{\tau} s \geqq e /(p-1)$. Then

$$
\eta^{p^{\nu}} \equiv 1 \bmod \mathfrak{p}^{s p^{\nu}}, \quad \eta^{p^{\nu}} \neq 1 \bmod \mathfrak{p}^{s p^{\nu+1}} \quad \text { for } \nu=0,1, \cdots, \tau
$$

and

$$
\eta^{p^{\nu}} \equiv 1 \bmod \mathfrak{p}^{s p \tau+(\nu-\tau) e} \quad \text { for } \nu \geqq \tau
$$

More precisely we have the following congruences by (1):

$$
\begin{aligned}
& \left(1+\gamma \pi^{s}\right)^{p^{\nu}} \\
& \equiv \begin{cases}1+\gamma^{p^{\nu}} \pi^{s p^{\nu}} \bmod \mathfrak{p}^{s p^{\nu+1}}, & \text { if } e /(p-1)<p^{\tau} s \text { and } 1 \leqq \nu \leqq \tau, \\
1+\gamma^{p \tau} p^{\nu-\tau} \pi^{s p^{\tau}} \bmod \mathfrak{p}^{s p^{\tau}(\nu-\tau) e+1}, & \text { if } e /(p-1)<p^{2} s \text { and } 1 \leqq \tau<\nu, \\
1+\gamma^{p^{\nu}} \pi^{s p^{\nu}} \bmod \mathfrak{p}^{s p^{\nu+1}}, & \text { if } e /(p-1)=p^{\tau} s \text { and } 1 \leqq \nu \leqq \tau, \\
1+\left(\gamma^{p^{\tau+1}}-\varepsilon \gamma^{p^{\tau}}\right) p^{\nu-\tau-1} \pi^{e+e_{1}} \bmod \mathfrak{p}^{(\nu-\tau) e+e_{1}+1}, & \text { if } e /(p-1)=p^{\tau} s \text { and } 0 \leqq \tau<\nu, \\
1+\gamma p^{\nu} \pi^{s} \bmod p^{\nu e+s+1}, & \text { if } e /(p-1)<s,\end{cases}
\end{aligned}
$$

where $\gamma$ is an integer of $k_{p}$.

LEMMA 9. Let $\eta_{i s}$ be principal units defined by Theorem A or Theorem $\mathrm{B}(1 \leqq i \leqq f, 1 \leqq s \leqq p e /(p-1), s \neq 0 \bmod p)$. Let $1 \leqq N<2 e+e_{1}$. Then we have for $\nu \geqq 1$

$$
\eta_{i s}^{p^{\nu}} \neq 1 \bmod \mathfrak{p}^{N+1}
$$

if and only if indices $i$ and $s$ satisfy the following conditions:

(i) $1 \leqq s \leqq N / p^{\nu}$, when $1 \leqq N<e+e_{1}$;

(ii) $1 \leqq s \leqq\left(e+e_{1}\right) / p^{\nu}$, but if $\mu \geqq 1$ and $\nu=\lambda$, then $(i, s) \neq\left(1, e_{0}\right)$, when $N=e+e_{1}$;

(iii) $1 \leqq s \leqq(N-e) / p^{\nu-1}$, but if $\nu=\lambda$ and $\lambda \geqq \nu\left(N: 1, e_{0}\right)$, then $(i, s)$ $\neq\left(1, e_{0}\right)$, when $e+e_{1}<N<2 e+e_{1}$ and $\mu \geqq 1$.

Proof. Let $\tau$ be the least non-negative integer such that 


$$
p^{\tau-1} s<e /(p-1) \leqq p^{\tau} s .
$$

Let $1 \leqq N<e+e_{1}$. If $1 \leqq s \leqq N / p^{\nu}$, then $\nu \leqq \tau$, otherwise it follows that $p^{\nu} s=p^{\tau} s \cdot p^{\nu-\tau} \geqq p e /(p-1) \geqq e+e_{1}>N$. Hence we see by Corollary 8 that $\eta_{i s}^{p \nu} \neq 1 \bmod p^{N+1}$. If $N / p^{\nu}<s$, then by Corollary 8 we have $\eta_{i s}^{p^{\nu}}$ $\equiv 1 \bmod \mathfrak{p}^{N+1}$.

Let $N=e+e_{1}$. If $1 \leqq s \leqq N / p^{\nu}$ and $p^{\tau-1} s<e /(p-1)<p^{\tau} s$, then $\nu \leqq \tau$. Hence by Corollary 8 we have $\eta_{i s}^{p^{\nu}} \neq 1 \bmod p^{N+1}$. If $e \equiv 0 \bmod (p-1)$, we put $e=\varphi\left(p^{\lambda}\right) e_{0},\left(e_{0}, p\right)=1$. If $1 \leqq s \leqq N / p^{\nu}$ and $p^{\tau} s=e /(p-1)$, then $\nu \leqq \tau+1$. In this case $s=e_{0}$ and $\tau=\lambda-1$, because of $s \neq 0 \bmod p$. If $\nu \leqq \tau=\lambda-1$, then by Corollary 8 we have $\eta_{i s}^{p^{\nu}}=\eta_{i e_{0}}^{p^{\nu}} \neq 1 \bmod \mathfrak{p}^{N+1}$. If $\nu=\tau+1=\lambda$, then we observe by Corollary 8 that

$$
\eta_{i s}^{p \nu}=\eta_{i e_{0}}^{p^{\lambda}} \equiv 1+\left(\omega_{i}^{p^{\lambda}}-\varepsilon \omega_{i}^{p^{\lambda-1}}\right) \pi^{e+e_{1}} \bmod \mathfrak{p}^{e+e_{1}+1} .
$$

If $\mu=0$, then $\eta_{i e_{0}}^{p^{\lambda}} \neq 1 \bmod p^{e+e_{1}+1}$, because of $\omega_{i}^{p^{\lambda}}-\varepsilon \omega_{i}^{p^{\lambda-1}} \neq 0 \bmod \mathfrak{p}$ (cf. (*) of $\S 4$ ). If $\mu \geqq 1$, then by Theorem $B$ we have

$$
\eta_{1 e_{0}}^{p^{2}} \equiv 1 \bmod p^{e+e_{1}+1}, \quad \eta_{i e_{0}}^{p^{2}} \neq 1 \bmod p^{e+e_{1}+1} \quad \text { for } i \neq 1 .
$$

Suppose that $\left(e+e_{1}\right) / p^{\nu}<s \leqq e+e_{1}=N$. If $0<\nu \leqq \tau$, then by Corollary 8 we get $\eta_{i s}^{p^{\nu}} \equiv 1 \bmod p^{N+1}$. If $p^{\tau} s>e /(p-1)$ and $0 \leqq \tau<\nu$, then $\eta_{i s}^{p^{\nu}}$ $\equiv 1 \bmod p^{N+1}$. If $p^{\tau} s=e /(p-1)$, then $s=e_{0}$ and $\tau=\lambda-1$. By the inequality $\left(e+e_{1}\right) / p^{\nu}<s=e_{0}=e_{1} / p^{\lambda-1}$, it follows $\nu>\lambda$. Hence $\eta_{i e_{0}}^{p^{\nu}}$ $\equiv 1 \bmod \mathfrak{p}^{N+1}$.

Let $e+e_{1}<N<2 e+e_{1}$ and assume $\mu \geqq 1$. If $1 \leqq s \leqq(N-e) / p^{\nu-1}$, then $\nu \leqq \tau+1$, otherwise $p^{\nu-1} s=p^{2} s \cdot p^{\nu-\tau-1} \geqq p e /(p-1)=e+e_{1}>N-e$. If $e /(p-1)<p^{s} s$ and $s \leqq(N-e) / p^{\nu-1}$, then by Corollary 8 we have $\eta_{i s}^{p^{\nu}} \neq 1 \bmod \mathfrak{p}^{N+1}$. If $e /(p-1)=p^{\tau} s$ and $\nu \leqq \tau$, then $\eta_{i s}^{p^{\nu}} \neq 1 \bmod \mathfrak{p}^{N+1}$. If $e /(p-1)=p^{\tau} s$ and $\nu=\tau+1$, then $s=e_{0}$ and $\tau=\lambda-1$. In this case we see by Theorem $\mathrm{B}$ that $\eta_{i e_{0}}^{p^{\nu}} \neq 1 \bmod \mathfrak{p}^{N+1}$ for $i \neq 1$. On the other hand we have for $i=1$

$$
\eta_{1 e_{0}}^{p^{\nu}} \equiv \begin{cases}1+\omega_{1}^{p^{\nu}} \pi^{e_{0} p^{\nu}} \bmod \mathfrak{p}^{e_{0} p^{\nu+1}} & \text { if } \nu \leqq \lambda-1, \\ 1+\left(\omega_{1}^{p^{\lambda}}-\varepsilon \omega_{1}^{p^{\lambda-1}}\right) p^{\nu-\lambda} \pi^{e+e_{1}} \bmod \mathfrak{p}^{(\nu-\lambda+1) e+e_{1}+1}, & \text { if } \nu \geqq \lambda,\end{cases}
$$

where $\omega_{1}^{p^{\lambda}}-\varepsilon \omega_{1}^{p^{\lambda-1}} \equiv 0 \bmod \mathfrak{p}$. If $\nu \leqq \lambda-1$, then $\eta_{1 e_{0}}^{p^{\nu}} \neq 1 \bmod \mathfrak{p}^{N+1}$, and $e_{0} \leqq(N-e) / p^{\nu-1}$. If $\nu>\lambda$, then $\eta_{1 e_{0}}^{p^{\nu}} \equiv 1 \bmod p^{N+1}$ and $e_{0}>(N-e) / p^{\nu-1}$. If $\nu=\lambda$, it may happen that $\eta_{1 e_{0}}^{p^{\lambda}} \equiv 1 \bmod p^{N+1}$, namely $\lambda \geqq \nu\left(N: 1, e_{0}\right)$. Hence $\eta_{i s}^{p^{\nu}} \neq 1 \bmod p^{N+1}$, where $1 \leqq i \leqq f, 1 \leqq s \leqq(N-e) / p^{\nu-1}, s \neq 0 \bmod p$, 
but if $\nu=\lambda$ and $\lambda \geqq \nu\left(N: 1, e_{0}\right)$, then $(i, s) \neq\left(1, e_{0}\right)$. Finally, suppose $(N-e) / p^{\nu-1}<s \leqq e+e_{1}$, where $e+e_{1}<N<2 e+e_{1}$. It then follows that $\nu \geqq \tau+1$. If $e /(p-1)<p^{\tau} s$, then $\eta_{i s}^{p \nu} \equiv 1 \bmod \mathfrak{p}^{N+1}$, because $s p^{\tau}$ $+(\nu-\tau) e>e_{1}+2 e>N$, if $\tau \leqq \nu-2 ; s p^{\tau}+(\nu-\tau) e=s p^{\nu-1}+e>N$, if $\tau=\nu-1$. If $e /(p-1)=p^{\tau} s$, then $s=e_{0}$ and $\tau=\lambda-1$. By the inequality $(N-e) / p^{\nu-1}<s=e_{0}=e_{1} / p^{\lambda-1}$ we have $\nu>\lambda$ and then $\eta_{i e_{0}}^{p^{\nu}}$ $\equiv 1 \bmod \mathfrak{p}^{N+1}$. If $s>e /(p-1)$ and $(N-e) / p^{\nu-1}<s$, then $\eta_{i s}^{p^{\nu}} \equiv 1 \bmod \mathfrak{p}^{N+1}$.

Thus Lemma 9 is proved.

q.e.d.

COROLlaRY 10. Suppose $\mu \geqq 1$. Let $\eta_{i s}$ and $\eta_{*}$ be principal units of Theorem $B$. Let ae $+e_{1} \leqq N<(a+1) e+e_{1}$ and $1 \leqq a \leqq \mu$. Then we have

$$
\begin{array}{ll}
\eta_{i s}^{p^{\nu}} \neq 1 \bmod \mathfrak{p}^{N+1}, & \eta_{*}^{p^{\nu}} \neq 1 \bmod \mathfrak{p}^{N+1}
\end{array}
$$

if and only if indices $i$ and $s$ satisfy the following conditions:

For $\nu \leqq a-1, \quad 1 \leqq s \leqq e+e_{1}$.

For $\nu \geqq a, \quad 1 \leqq s \leqq(N-(a+\delta-1) e) / p^{\nu-a-\delta+1}$,

but if $\nu\left(N: 1, e_{0}\right) \leqq \nu \leqq \lambda+a-1$, then $(i, s) \neq\left(1, e_{0}\right)$,

where

$$
\delta= \begin{cases}0, & \text { if } N=a e+e_{1}, \\ 1, & \text { if } a e+e_{1}<N<(a+1) e+e_{1} .\end{cases}
$$

Proof. Let $N=a e+e_{1}$. It is obvious by Proposition 6 that $H_{e+e_{1}+1}^{p^{a-1}} \cong H_{N+1}$. Since we have $\eta_{i s} \neq 1 \bmod \mathfrak{p}^{e+e_{1}+1}\left(1 \leqq s \leqq e+e_{1}\right)$ and $\eta_{*}$ $\neq 1 \bmod \mathfrak{p}^{e+e_{1}+1}, \eta_{i s}^{p^{\nu}} \neq 1 \bmod \mathfrak{p}^{N+1}$ and $\eta_{*}^{p^{\nu}} \neq 1 \bmod \mathfrak{p}^{N+1}$ for $\nu \leqq a-1$. Let $(i, s) \neq\left(1, e_{0}\right)$ and $\nu \geqq a$. By Lemma 9 we find that $\eta_{i s}^{p^{\nu}} \neq 1 \bmod \mathfrak{p}^{e+e_{1}+1}$ for $1 \leqq s \leqq\left(e+e_{1}\right) / p^{\nu}$. Hence it follows that $\eta_{i s}^{p^{\nu+a-1}} \neq 1 \bmod \mathfrak{p}^{N+1}$ for $1 \leqq s$ $\leqq\left(e+e_{1}\right) / p^{\nu}$. Moreover, since $H_{e+e_{1}+1}^{p^{a-1}} \cong H_{N+1}$, we see that $\eta_{i s}^{p^{\nu}} \neq 1 \bmod \mathfrak{p}^{N+1}$ for $1 \leqq s \leqq\left(e+e_{1}\right) / p^{\nu-a+1}$. Let $(i, s)=\left(1, e_{0}\right)$. Then $e_{0}=e_{1} / p^{\lambda-1}$ $\leqq\left(e+e_{1}\right) / p^{\nu-a+1}=e_{1} / p^{\nu-a}$ if and only if $\nu \leqq \lambda+a-1$. By Corollary 8 we have $\eta_{1 e_{0}}^{p^{\lambda}} \equiv 1 \bmod \mathfrak{p}^{e+e_{1}+1}$ and hence $\eta_{1 e_{0}}^{p^{\lambda+a-1}} \equiv 1 \bmod \mathfrak{p}^{N+1}$, that is, $\lambda$ $\leqq \nu\left(N: 1, e_{0}\right) \leqq \lambda+a-1$.

Since $\eta_{*} \equiv 1 \bmod \mathfrak{p}^{e+e_{1}}, \eta_{*} \neq 1 \bmod \mathfrak{p}^{e+e_{1}+1}$, we have $\eta_{*}^{p^{\nu}} \equiv 1 \bmod \mathfrak{p}^{(\nu+1) e+e_{1}}$, $\eta_{*}^{p^{\nu}} \neq 1 \bmod \mathfrak{p}^{(\nu+1) e+e_{1}+1}$ for $\nu=0,1, \cdots$.

Let $a e+e_{1}<N<(a+1) e+e_{1}$. It then follows from Proposition 6 that $H_{N-(a-1) e+1}^{p^{a-1}} \cong H_{N+1}$. Hence by the same arguments as above we have 
the latter half of Corollary 10. We note that $\lambda \leqq \nu\left(N: 1, e_{0}\right) \leqq \lambda+a$.

q.e.d.

From Lemma 9 and Corollary 10, the numbers $g_{N}(\nu)$, exponents $\nu(N: i, s)$ and $\nu(N: *)$ defined in $\S 2$ are given as follows:

If $1 \leqq N<e+e_{1}$, or if $\mu=0$ and $N=e+e_{1}$, then

$$
g_{N}(\nu)=\left(N-\left[\frac{N}{p}\right]-\left[\frac{N}{p^{\nu}}\right]+\left[\frac{N}{p^{\nu+1}}\right]\right) f, \quad(\nu \geqq 1),
$$

and

$$
\nu(N: i, s)=\nu \quad \text { for } N / p^{\nu}<s \leqq N / p^{\nu-1},
$$

where $1 \leqq i \leqq f, 1 \leqq s \leqq N$ and $s \neq 0 \bmod p$.

If $\mu \geqq 1$ and $a e+e_{1} \leqq N<(a+1) e+e_{1}(1 \leqq a \leqq \mu)$, then

$$
\left\{\begin{aligned}
g_{N}(\nu)= & 0, \\
g_{N}(\nu)= & \left(e+e_{1}-\left[\frac{e+e_{1}}{p}\right]-\left[\frac{N-(a+\delta-1) e}{p^{\nu-a-\delta+1}}\right]\right. \\
& \left.+\left[\frac{N-(a+\delta-1) e}{p^{\nu-a-\delta+2}}\right]\right) f+\bar{g}_{N}(\nu), \quad \text { for } \nu \geqq a,
\end{aligned}\right.
$$

where

$$
\bar{g}_{N}(\nu)= \begin{cases}2, & \text { if } \nu\left(N: 1, e_{0}\right) \leqq \nu \leqq \lambda+a-1 \\ 1, & \text { otherwise }\end{cases}
$$

and

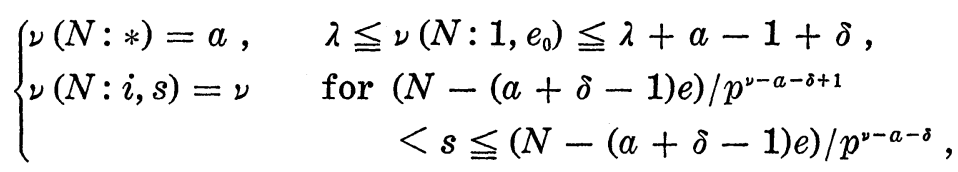

where $1 \leqq i \leqq f, 1 \leqq s \leqq e+e_{1}, s \neq 0 \bmod p,(i, s) \neq\left(1, e_{0}\right)$ and $\delta$ is given by Corollary 10. We note that if $\lambda=\mu$, or $N=e+e_{1}$, then $\nu\left(N: 1, e_{0}\right)$ $=\lambda$.

It then follows from (13) and (15) that

$$
\begin{aligned}
g_{N}(1)+ & \sum_{\nu=2}^{\infty} \nu\left(g_{N}(\nu)-g_{N}(\nu-1)\right) \\
& = \begin{cases}N f, & \text { if } 1 \leqq N \leqq e+e_{1}, \\
N f+\nu\left(N: 1, e_{0}\right)-\lambda, & \text { if } a e+e_{1} \leqq N<(a+1) e+e_{1}\end{cases}
\end{aligned}
$$


Thus (2) or $\left(8_{1}\right)$ is a basis of $H_{1} / H_{N+1}$ and $\left(8_{2}\right)$ is a basis of $H_{1} / H_{N+1}$ if and only if $\nu\left(N: 1, e_{0}\right)=\lambda$.

Now we establish a basis of $H_{N+1}$.

Proposition 11. (A). Suppose that $\mu=0$. It then follows that for each $t \geqq 0$ and $1 \leqq N \leqq e+e_{1}$

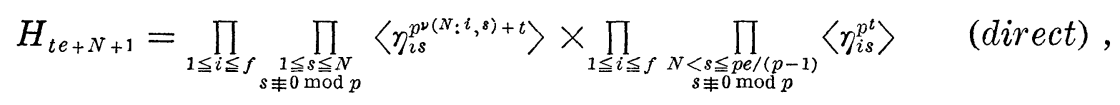

where $\eta_{i s}$ are principal units of Theorem $\mathrm{A}$ and $\nu(N: i, s)$ are given by (14).

(B). Suppose $\mu \geqq 1$. Let ae $+e_{1} \leqq N<(a+1) e+e_{1}$ and $1 \leqq a \leqq \mu$. Then it follows that for each $t \geqq 0$

$$
H_{t e+N+1}=\left\langle\eta_{*}^{p^{a+t}}\right\rangle \times \prod_{(i, s) \in S}\left\langle\eta_{i s}^{p \nu(N: i, s)+\iota}\right\rangle \quad \text { (direct) },
$$

where $\eta_{*}, \eta_{i s}$ are principal units of Theorem $\mathrm{B}, \nu(N: i, s)$ are given by (16) and $S$ is the set defined by (5).

Proof. We first notice that by Theorem A or (7) multiplicative expressions described as above are surely direct products.

(A). Suppose that $\mu=0$ and $1 \leqq N \leqq e+e_{1}$. Put

$$
H_{N+1}^{\prime}=\prod_{1 \leqq i \leqq f} \prod_{\substack{1 \leqq s \leqq N \\ s \neq 0 \text { mod } p}}\left\langle\eta_{i s}^{p \nu(N: i, s)}\right\rangle \times \prod_{1 \leqq i \leqq f} \prod_{\substack{N<s \leq p e /(p-1) \\ s \neq 0 \text { mod } p}}\left\langle\eta_{i s}\right\rangle \quad \text { (direct). }
$$

Then $H_{N+1}^{\prime}$ is a subgroup of $H_{N+1}$. It is proved that $H_{N+1}^{\prime}=H_{N+1}$. Indeed,

$$
\left(H_{1}: H_{N+1}^{\prime}\right)=\prod_{1 \leqq i \leqq f} \prod_{\substack{1 \leq s \leq N \\ s \neq 0 \text { mod } p}} p^{\nu(N: i, s)} ;
$$

from (13) and (17) we have

$$
\sum_{1 \leqq i \leqq f} \sum_{\substack{0 \leq s \leq N \\ s \neq 0 \bmod p}} \nu(N: i, s)=g_{N}(1)+\sum_{\nu=2}^{\infty} \nu\left(g_{N}(\nu)-g_{N}(\nu-1)\right)=N f .
$$

Hence we have $\left(H_{1}: H_{N+1}^{\prime}\right)=p^{N f}=\left(H_{1}: H_{N+1}\right)$, as was to be shown.

If $e_{1} \leqq N \leqq e+e_{1}$, then we observe by Proposition 6 that $H_{N+1}^{p^{t}}$ $\cong H_{t e+N+1}$ for each $t \geqq 0$. Therefore, we have the direct decomposition of $H_{t e+N+1}$.

(B). Suppose $\mu \geqq 1$. Let $a e+e_{1} \leqq N<(a+1) e+e_{1}$ and $1 \leqq a \leqq \mu$. 
Put

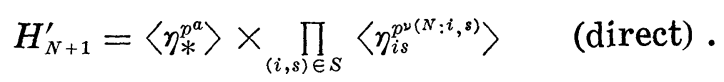

Then $H_{N+1}^{\prime}$ is a subgroup of $H_{N+1}$ and $H_{01}$. We contend $H_{N+1}^{\prime}=H_{N+1}$. Indeed, since we have $\left(H_{1}: H_{01}\right)=p^{2}$ by $[2, \mathrm{p} .231]$,

$$
\left(H_{1}: H_{N+1}^{\prime}\right)=\left(H_{1}: H_{01}\right)\left(H_{01}: H_{N+1}^{\prime}\right)=p^{\lambda} p^{a} \prod_{(i, s) \in S} p^{\nu(N: i, s)} ;
$$

it follows from (15), (16) and (17) that

$$
\begin{aligned}
& \sum_{(i, s) \in S} \nu(N: i, s) \\
&= a\left(g_{N}(a)-1\right)+\sum_{\nu=a+1}^{\nu\left(N: 1, e_{0}\right)-1} \nu\left\{\left(g_{N}(\nu)-1\right)-\left(g_{N}(\nu-1)-1\right)\right\} \\
&+\nu\left(N: 1, e_{0}\right)\left\{\left(g_{N}\left(\nu\left(N: 1, e_{0}\right)\right)-2\right)-\left(g_{N}\left(\nu\left(N: 1, e_{0}\right)-1\right)-1\right)\right\} \\
&+\sum_{\nu=\nu\left(N: 1, e_{0}\right)+1}^{\infty} \nu\left\{\left(g_{N}(\nu)-2\right)-\left(g_{N}(\nu-1)-2\right)\right\} \\
&= a g_{N}(a)+\sum_{\nu=a+1}^{\infty} \nu\left(g_{N}(\nu)-g_{N}(\nu-1)\right)-a-\nu\left(N: 1, e_{0}\right) \\
&= N f-(\lambda+a) .
\end{aligned}
$$

Hence we get $\left(H_{1}: H_{N+1}^{\prime}\right)=p^{N f}=\left(H_{1}: H_{N+1}\right)$, as desired.

Finally it is clear that $H_{t e+N+1} \cong H_{N+1}^{p^{t}}$ for each $t \geqq 0$ by Proposition

6. Thus we have the direct decomposition of $H_{t e+N+1}$. q.e.d.

\section{§6. Proof of Theorem 2 and Theorem 3}

From Theorem A, Proposition 11, (4) and (13) we have Theorem 2 .

Now we shall prove Theorem 3 . Suppose that $k_{p}$ contains $\zeta_{\mu}(\mu \geqq 1)$, but does not contain $\zeta_{\mu+1}$.

( I ). In the case where $1 \leqq N<e+e_{1}$, it is verified by (17) that $\left(8_{1}\right)$ is a basis of $H_{1} / H_{N+1}$. Hence the direct decomposition of $G\left(\mathfrak{p}^{N+1}\right)$ is obtained by (4), (13) and (14).

(II). In the case where $e+e_{1} \leqq N<(\mu+1) e+e_{1}$ and $\nu\left(N: 1, e_{0}\right)$ $=\lambda$, we know by (17) that $\left(8_{2}\right)$ is a basis of $H_{1} / H_{N+1}$. Hence the direct decomposition of $G\left(p^{N+1}\right)$ is obtained by (4), (15) and (16).

(III). In the case where $e+e_{1}<N<(\mu+1) e+e_{1}$ and $\nu\left(N: 1, e_{0}\right)$ $>\lambda$, we see by Proposition 11 and (7) that $\eta_{*}, \eta_{i s}((i, s) \in S)$ are independent modulo $\mathfrak{p}^{N+1}$, that is, $\eta_{*}^{x_{*}}$. $\prod_{(i, s) \in S} \eta_{i s}^{x_{i s}} \equiv 1 \bmod \mathfrak{p}^{N+1}$ if and only if $x_{*} \equiv 0 \bmod p^{a}$ ani $x_{i s} \equiv 0 \bmod p^{\nu(N: i, s)}$ for all $(i, s) \in S$.

From the relation (6) we have a congruence 


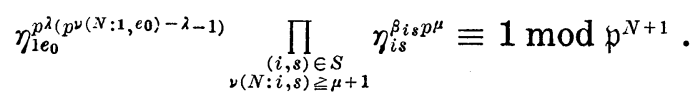

Since $\left(H_{1}: H_{01}\right)=p^{2}$ and $H_{N+1}$ is a subgroup of $H_{01}, p^{2}$ is the least positive integer such that $\eta_{1 e_{0}}^{p^{2}} \equiv \eta_{0} \bmod p^{N+1}$ for some $\eta_{0} \in H_{01}$. Hence the structure of $H_{1} / H_{N+1}$ having a system of canonical generators $\left(8_{2}\right)$ is determined by (18) only. We put

$$
\begin{array}{ll}
\beta_{*} p^{\mu}=\beta_{*}^{\prime} p^{a_{*}}, & \left(\beta_{*}^{\prime}, p\right)=1, \\
\beta_{i s} p^{\mu}=\beta_{i s}^{\prime} p^{a_{i s}}, & \left(\beta_{i s}^{\prime}, p\right)=1
\end{array} \quad \text { for }(i, s) \in S .
$$

It is then clear that instead of $\left(8_{2}\right)$

$$
\left\{\eta_{i e_{0}}^{\left.p N_{0}: 1, e 0\right)-2-1} H_{N+1}, \eta_{*}^{\beta^{*}} H_{N+1}, \eta_{i s}^{\beta^{\prime} s} H_{N+1}\right\}_{(i, s) \in S}
$$

is also a system of canonical generators for $H_{1} / H_{N+1}$.

Let $M$, a free $Z$-module, and $\psi: M \rightarrow H_{1} / H_{N+1}$ be as defined in $\S 2$. Put

$$
a_{i s}=\min \left\{\nu(N: i, s), a_{i s}^{\prime}\right\} \quad \text { for }(i, s) \in S .
$$

Then from Proposition 11 and by (18) a system of canonical generators for $\operatorname{Ker} \psi$ is given by

$$
\left\{p^{a} \tilde{\eta}_{*}, p^{\nu\left(N .1, e_{0}\right)} \tilde{\eta}_{1 e_{0}}, p^{\nu(N: i, s)} \tilde{\eta}_{i s}, p^{2} \tilde{\eta}_{1 e_{0}}+\sum_{(i, s) \in S} p^{a_{i s}} \tilde{\eta}_{i s}\right\},
$$

where $(i, s) \in S$. Then the rank of $\operatorname{Ker} \psi$ is equal to $(e f+1)$ because the rank of $H_{1} / H_{N+1}$ is equal to $(e f+1)$ from Theorem 1 . The direct decomposition of $H_{1} / H_{N+1} \cong M / \mathrm{Ker} \psi$ is determined by elementary divisors of the matrix (9) of Theorem 3. Thus (III) of Theorem 3 is proved.

Finally, (IV) of Theorem 3 is trivially obtained from Lemma 7 . Thus Theorem 3 is completely proved.

\section{§ 7. Proof of Corollary 4}

Let $\mathfrak{p}$ be an unramified prime ideal of $k$, lying above a rational prime $p$. Assume that $p$ is odd. Then by Theorem 2 we observe that $b_{1}(1)=f$ and $b_{1}(\nu)=0$ for $\nu \geqq 2$. Let $p=2$. Then $e=e_{1}=1$ and $\lambda=\mu=1$. Therefore, we have by (I) and (II) of Theorem 3

$$
\begin{array}{lll}
b_{1}(1)=f, & b_{1}(\nu)=0 & \text { for } \nu \geqq 2, \\
b_{2}(1)=2, & b_{2}(2)=f-1, \quad b_{2}(\nu)=0 & \text { for } \nu \geqq 3 .
\end{array}
$$


Thus Corollary 4 is obtained from Theorem 2 and Theorem 3.

\section{§ 8. Supplement to Theorem 3}

We assume that $k_{\mathfrak{p}}$ contains $\zeta_{\mu}(\mu \geqq 1)$ but does not contain $\zeta_{\mu+1}$. Suppose that $\lambda>\mu \geqq 1$ and $a e+e_{1} \leqq N<(a+1) e+e_{1}(1 \leqq a \leqq \mu)$. In this section we shall prove that if one of exponents $\nu(N: i, s)$ satisfies a certain condition, then the direct decomposition of $H_{1} / H_{N+1}$ is induced by that of $H_{1} / H_{N-e+1}$.

If $\lambda>\mu \geqq 1$, then a $Z_{p}$-basis of $H_{1}$ is given as follows (cf. [2, p. 232233]). Let $H_{01}$ be the free $Z_{p}$-group of $H_{1}$ defined by (7). By (6) we observe that $\eta_{1 e_{0}}^{p^{\lambda-\mu} \zeta_{\mu}^{-1}}$ does not belong to $H_{01}^{p}=\left\{\eta_{0}^{p} \mid \eta_{0} \in H_{01}\right\}$. There exists $\beta_{i_{0} s_{0}}$ such that $\beta_{i_{0} s_{0}}$ is prime to $p$. If $\beta_{*}$ is prime to $p$, we may take $\beta_{i_{0} s_{0}}=\beta_{*}$. Hence $\eta_{i_{0} s_{0}}$ can be written in the form

$$
\eta_{i_{0} s_{0}}=\zeta_{\mu}^{\alpha \mu} \prod_{\substack{(i, s) \in S^{\prime} \\(i, s) \neq\left(i_{0}, s_{0}\right)}} \eta_{i s}^{\alpha_{i} s} \cdot \eta_{1 e_{0}}^{p^{2-\mu_{\alpha}}}
$$

where $S^{\prime}=S \cup\{*\}, \alpha_{\mu}$ is a rational integer, prime to $p\left(1 \leqq \alpha_{\mu}<p^{\mu}\right), \alpha_{i s}$ are $p$-adic integers and $\alpha_{1 e_{0}}$ is a $p$-adic integer, prime to $p$ (cf. [2, II in p. 209]). We then have a $Z_{p}$-free part $\tilde{H}_{01}$ of $H_{1}$, expressed as direct product:

$$
\tilde{H}_{01}=\prod_{\substack{(i, s) \in S^{\prime} \\(i, s) \neq\left(i_{0}, s_{0}\right)}}\left\langle\eta_{i s}\right\rangle \times\left\langle\eta_{1 e_{0}}\right\rangle \quad \text { (direct) }
$$

From Proposition 6 we find that $H_{N-e+1}^{p} \cong H_{N+1}$, where $a e+e_{1} \leqq N$ $<(a+1) e+e_{1}$ and $1 \leqq a \leqq \mu$. Therefore by Proposition 11 we have

$$
H_{N-e+1}=\left\langle\eta_{*}^{p^{a-1}}\right\rangle \times \prod_{(i, s) \in S}\left\langle\eta_{i s}^{\left.p^{\nu(N: i, s)-1}\right\rangle} \quad\right. \text { (direct) . }
$$

It then follows from (19) that $H_{N-e+1}$ is a subgroup of $\tilde{H}_{01}$ if and only if $\nu\left(N: i_{0}, s_{0}\right)-1 \geqq \mu$. We note that $\nu(N: *)=a\langle\mu+1$ (see (16)). If $\nu\left(N: i_{0}, s_{0}\right) \geqq \mu+1$, one see also that

$$
H_{1} / H_{N-e+1} \cong\left\langle\zeta_{\mu}\right\rangle \times \tilde{H}_{01} / H_{N-e+1} \quad \text { (direct) . }
$$

The direct decomposition of $G\left(p^{N-e+1}\right)$ is obtained from (I) $\sim$ (III) of Theorem 3 and by Lemma 7 , say of type $\left(p^{f}-1, p^{\mu}, p^{c_{1}^{\prime}}, \cdots, p^{c_{e}^{\prime} f}\right)$. Then $G\left(\mathfrak{p}^{N+1}\right)$ is of type $\left(p^{f}-1, p^{\mu}, p^{c_{1}^{\prime+1}}, \cdots, p^{c^{\prime} f+1}\right)$ by Lemma 7 . 


\section{§9. Examples}

(i). Let $p$ be an odd prime and $\zeta_{1}$ be a primitive $p$-th root of unity. Put $k=\boldsymbol{Q}\left(\zeta_{1}\right)$ and $\mathfrak{p}=\left(1-\zeta_{1}\right)$. Then we have an expression of $G\left(\mathfrak{p}^{N+1}\right)$ as direct product for each $t \geqq 0$ :

$$
\begin{aligned}
& G\left(\mathfrak{p}^{N+1}\right)
\end{aligned}
$$

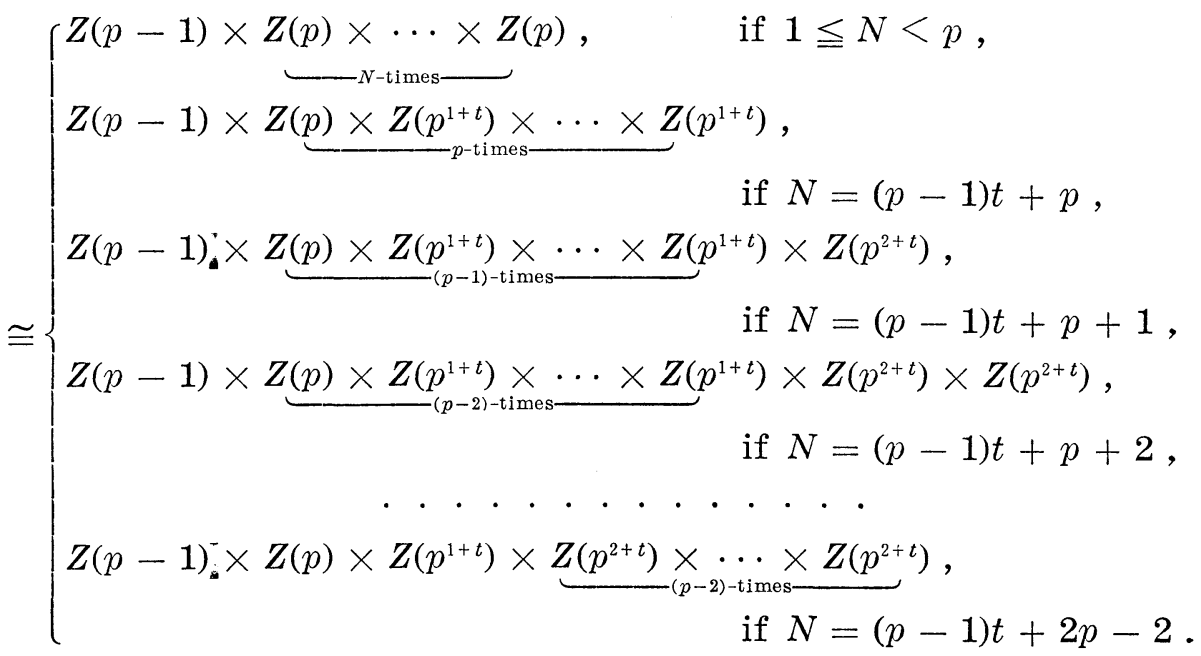

(ii). Let $d$ be a square free rational integer such that $d \equiv 2 \bmod 4$. Put $k=\boldsymbol{Q}(\sqrt{d})$ and let $\mathfrak{p}$ be a prime ideal of $k$, lying above 2 . Then $e=e_{1}=2, \lambda=2$ and $\mu=1$. By (I) of Theorem 3 we have

$$
G\left(\mathfrak{p}^{2}\right) \cong Z(2), \quad G\left(\mathfrak{p}^{3}\right) \cong Z\left(2^{2}\right), \quad G\left(\mathfrak{p}^{4}\right) \cong Z(2) \times Z\left(2^{2}\right) .
$$

By [4] we see that for $N=e+e_{1}=4, \nu(4: 1,1)=2=\lambda>\mu$. Hence for each $t \geqq 0$ we obtain by (II) and (IV) of Theorem 3

$$
G\left(\mathfrak{p}^{2 t+5}\right) \cong Z(2) \times Z\left(2^{1+t}\right) \times Z\left(2^{2+t}\right) .
$$

Furthermore, it is shown in [4] that $-\eta_{11}^{2} \equiv \eta_{13} \bmod \mathfrak{p}^{4}$. It then follows that for $N=5\left(e+e_{1}<N<2 e+e_{1}\right), \nu(5: 1,1)=3>\lambda$ and $\nu(5: 1,3)$ $=2=\lambda>\mu$. Hence from the arguments of $\S 8$ we see that $H_{4}$ is a subgroup of the free part of $H_{1}$. From the result of $\S 8$ and by Theorem 1 the direct decomposition of $G\left(p^{6}\right)$ is induced by that of $G\left(p^{4}\right)$, that is, expressed as follows:

$$
G\left(\mathfrak{p}^{6}\right) \cong Z(2) \times Z(2) \times Z\left(2^{3}\right) .
$$

Therefore, we see by (IV) of Theorem 3 that for each $t \geqq 0$ 


$$
G\left(\mathfrak{p}^{2 \ell+6}\right) \cong Z(2) \times Z\left(2^{1+\imath}\right) \times Z\left(2^{3+\ell}\right)
$$

For $N=5$ the matrix (9) of Theorem 3 is equal to

$$
\left(\begin{array}{lll}
2 & 0 & 0 \\
0 & 2^{2} & 0 \\
0 & 0 & 2^{3} \\
2 & 2 & 2^{2}
\end{array}\right\}
$$

It is then clear that

$$
\left(\begin{array}{rrrr}
1 & 0 & 0 & 0 \\
-1 & 0 & 0 & 1 \\
0 & 0 & 1 & 0 \\
2 & 1 & 1 & -2
\end{array}\right)\left(\begin{array}{lll}
2 & 0 & 0 \\
0 & 2^{2} & 0 \\
0 & 0 & 2^{3} \\
2 & 2 & 2^{2}
\end{array}\right)\left(\begin{array}{lll}
1 & 0 & 0 \\
0 & 1 & -2 \\
0 & 0 & 1
\end{array}\right)=\left(\begin{array}{lll}
2 & 0 & 0 \\
0 & 2 & 0 \\
0 & 0 & 2^{3} \\
0 & 0 & 0
\end{array}\right),
$$

which shows the direct decomposition of $H_{1} / H_{6}$, too.

\section{BIBLIOGRAPHY}

[1] Albis Gonzalez, V. S., A remark on primitive root and ramification, Rev. Columbiana Math., 7 (1973), 93-98.

[ 2 ] Hasse, H., Zahlentheorie, Akademie-Verlag, Berlin, 2 Aufl., 1963.

[ 3 ] — - Bericht über neuere Untersuchungen und Probleme aus der Theorie der algebraischen Zahlkörper, Physica Verlag, 1965.

[4] H.-Koch, F., Einseinheitengruppen und prime Restklassengruppen in quadratischen Zahlkörper, J. Number Theory, 4 (1972), 70-77.

[5] Narkiewicz, W., Elementary and analytic theory of algebraic numbers, Monogr. Math., 57, PWN-Polish Sci. Publishers, 1974.

[6 ] Ranum, A., The group of classes of congruent quadratic integers with respect to composite ideal modulus, Trans. Amer. Math. Soc., 11 (1910), 172-198.

[ 7 ] Serre, J.-P., Sur les corps locaux à corps résiduel algébriquement clos, Bull. Soc. Math. France, 89 (1961), 105-154.

[8] Takenouchi, T., On the classes of congruent integers in an algebraic körper, J. College of Sci. Tokyo Imp. Univ., XXXVI, Article I (1913), 1-28.

[ 9 ] Wyman, B. F., Wildly ramified Gamma extensions, Amer. J. Math., 91 (1969), $135-152$.

Department of Mathematics

College of Liberal Arts

Toyama University 